\title{
Applications of a salt tolerant cation exchanger carrying sulfate
}

\section{groups}

\author{
Egbert Müller ${ }^{1}$, Djuro Josic ${ }^{2}$, Marija Begic $^{3}$, Suzana Pecenkovic $^{4}$, and Uros Andjelkovic ${ }^{5}$ \\ ${ }^{1}$ Tosoh Bioscience GmbH \\ ${ }^{2}$ Warren Alpert Medical School of Brown University \\ ${ }^{3}$ Juraj Dobrila University of Pula \\ ${ }^{4}$ General Hospital Pula \\ ${ }^{5}$ Affiliation not available
}

February 24, 2021

\begin{abstract}
Difference between two strong cation-exchange resins, namely sulfonium type and sulfate type regarding both their salt tolerance and hydrophobicity were investigated. There is only tiny variation between sulfate and sulfonic group and at the first glance it seems unlikely that it could be the reason for changed selectivity and salt tolerance that was detected in our preliminary experiments. For that reason salt tolerance and hydrophobicity of both ligands was investigated by using two representative polymethacrylate-based ion exchangers as for the sulfonium type TOYOPEARL GigaCap S-650M and for the sulfate type TOYOPEARL Sulfate-650F. In addition some in-silico calculations were performed for model substances representing the sulfonium and sulfate group, and significant differences were calculated regarding their hydrophobicity. These experiments confirmed the working hypothesis that salt tolerance and higher affinity and selectivity for some human plasma derived vitamin $\mathrm{K}$ dependent clotting factors and inhibitors are interrelated and dependent from the presence of the sulfate group. The affinity for these proteins was experimentally verified by separation of clotting factor IX from the prothrombin complex concentrate. Presented results show that a simple and fast separation between clotting factor IX and other vitamin K dependent clotting factors II, VII and X is possible, only if the resin with the sulfate, and not with sulfonic acid ligand was applied. Consequently, an immediate application of undiluted feedstock or the eluate from previous isolation step to sulfate resin is possible, and a significant optimization of downstream process can be achieved.
\end{abstract}

\section{Hosted file}

ManuscriptS04to submit Version final letzte Version.pdf available at https://authorea.com/ users/397963/articles/510684-applications-of-a-salt-tolerant-cation-exchanger-carryingsulfate-groups 